\title{
EVIDENCE FOR A POWER LAW INTENSITY CODE IN THE COUPLED CONES OF THE TURTLE
}

\author{
Vincent Pluvinage and Daniel G. Green* \\ From the Vision Research Laboratory, Neuroscience Building, University of Michigan, \\ 1103 E. Huron, Ann Arbor, MI 48109, U.S.A.
}

(Received 3 November 1988; in revised form 22 September 1989)

\begin{abstract}
The hyperpolarizing responses to light were recorded intracellularly from red cones of the turtle, Pseudemys scripta elegans. Pairs of slit stimuli were flashed alone or together at various intensities, one slit positioned on the receptive field center and the other displaced $30 \mu \mathrm{m}$. The peak amplitude of the response was measured, and the results analyzed to quantify the relationship between the light intensity and the size of the neural signal evoked prior to the spatial interactions occurring in the network of coupled cones. This signal, $E$, was found to be described by a compressive power law, $E=k \cdot I^{0.5}$, where $I$ is slit intensity. Evidence that the inferred excitation function describes a local mechanism independent of the slit position was obtained by measuring the response and the sensitivity receptive field profiles. The response and the sensitivity fields both decreased exponentially, but with space constants that differed by a factor of two, indicating in still another way the existence of an early square-root transformation.
\end{abstract}

Retinal cone Phototransduction Photoreceptor

\section{INTRODUCTION}

Power law relationships which describe how visual experience varies with the magnitude of the physical stimulus (Stevens, 1970) have only occasionally been found to describe the relationship between the responses of retinal cells and stimulus intensity (Easter, 1968; Stone \& Fabian, 1968; Grusser, 1971; Levine \& Abramov, 1975; Enroth-Cugell \& Harding, 1980). The physiological experiments which suggest power law relationships have three things in common: (a) they use a common methodology; and (b) they conclude that the exponential in the power law realtionship is about 0.5 ; and (c) they have been done on retinal ganglion cells. In this paper we show that the same methodology applied to turtle cones leads to the same conclusion (i.e. power law with 0.5 exponent). In a companion paper we show that the apparent square-root compress is due not to the relationship between stimulus intensity and the photovoltage in a single cone but arises out of local saturation and recruitment of scattered light responses.

In turtle cones the hyperpolarizing responses depend on both the intensity and the spatial extent of the light stimuli. At least two

-To whom correspondence should be addressed. physiological mechanisms are involved: a local transduction mechanism and a coupling network mediating lateral interactions between cones. In addition, horizontal cells feed back onto cones, but their effects are noticeable only when large and bright stimuli are used and are negligible for the type of stimuli we report here (Baylor, Fuortes \& O'Bryan, 1971; Baylor \& Hodgkin, 1973; Baylor, Hodgkin \& Lamb, 1974; Lamb \& Simon, 1976).

In order to measure how cones in a coupled network respond to variations in stimulus intensity we have applied a technique called additive conjoint measurement (Debreu, 1959; Kruskal, 1965; Krantz, Luce, Suppes \& Tversky, 1971; Krantz, 1973). The approach is similar in concept to the one used by Easter (1968) and others to study intensity coding at the ganglion cell level. When applied to cone response the technique theoretically allows one to infer the local intensity coding which occurs prior to the spatial interactions between coupled cones. This local intensity coding, which we call excitation, is not easily measurable in an intact retina. Even when using a small stimulus focused on the impaled cell the intracellularly recorded hyperpolarizing response does not provide a direct measurement of the excitation signal since light always scatters to adjacent coupled receptors. Nonlinearities such as voltage-dependent 
conductances can also intervene between the excitation and the recorded voltage response. Excitation inferred by conjoint measurement describes the input-output relationship of a transduction process which depends solely on the local light intensity, and is independent of the light falling on other parts of the receptive field. Thus, the aim of this approach is to dissect the mechanisms occurring before the spatial interactions from those which affect the pooled signals.

The data analysis is based on the following paradigm. We assume that when two narrow slits of light are flashed at different positions in the cone receptive field a light transduction mechanism generates at each position a neural signal (excitation) which is independent of the signal at the other position. The intracellular hyperpolarizing response to light is assumed to be a unique monotonically increasing function of the operationally defined excitation. For the moment the nature of the excitation will be left unspecified. It may help to think of excitation being the photocurrent generated in the cone outer segments, though as we show in the second paper it is not a photocurrent. Because the cones are coupled, when two slits are flashed simultaneously the signals evoked by the two slits summate in the impaled cone. By adjusting the intensity of a slit, one can vary the amplitude of the response it evokes until it matches the response evoked by one or more other slits. When the responses are matched, equal amounts of excitation signal are assumed to have been produced in the impaled cell, even though the cones are stimulated differently.

By systematically varying the intensity of the slits and matching responses one can infer how transduction converts light intensity into excitation. As we will explain, it should be possible to make this inference even though other processes intervene in generating the measured voltage response (see Methods for details). The experiments presented here show that, in turtle cones, the inferred early transformation is well described over two log-units of intensity by a compressive power law:

$$
E=k \cdot I^{m} ;
$$

where $I$ is the light intensity, $k$ is a constant, $E$ is the operationally-defined excitation variable and the exponent " $\mathrm{m}$ " is close to 0.5 (a "square root" law). This is consistent with the results obtained from ganglion cell studies (on goldfish:
Easter, 1968; Levine \& Abramov, 1975; on cat: Stone \& Fabian, 1968; Grusser, 1971; EnrothCugell \& Harding, 1980).

Our finding that an early "square root" transformation occurs at the photoreceptor level is very surprising and would seem to be in conflict with the Michaelis-Menten relationship found in direct photocurrent measurements (Schnapf \& McBurney, 1980) and full-field photovoltage measurements (Baylor \& Hodgkin, 1973) performed on turtle cones. The purpose of this paper is to document this new finding. The physiological mechanisms which produce the "square root" transformation are examined in a second paper (Pluvinage \& Green, 1990) where we show that our finding does not conflict with previous studies.

\section{METHODS}

\section{Preparation}

The experiments were performed on darkadapted retinas of the red-eared turtle, Pseudemys scripta elegans. After decapitation, the eye was removed and hemisected along a frontal plane. The eye cup was placed in a recording chamber attached to a $\mathrm{Ag} / \mathrm{AgCl}$ reference electrode with a drop of Ringer mixed with dissolved gelatin $(5 \% \mathrm{w} / \mathrm{v})$. The vitreous humor was drained away using little triangular pieces of tissue placed at the edge of the eye cup. A steady stream of moist oxygen was blown into the chamber.

\section{Recording}

The high impedance microelectrodes (200-800 M $\Omega$ ) were pulled from Omega Dot glass capillary tubing on either a Livingston puller or a Sutter puller (Brown \& Flaming, $1977)$ and filled with potassium acetate (2 M, pH 7).

To ensure that the light stimuli were in best focus on the photoreceptor layer, the following procedure was used. The stimulus was focused on the surface of the water contained in a reservoir on the cover of the experimental chamber. The microelectrode was lowered until it just touched the surface at the center of the stimulus. The depth counter of the modified Kopf microdrive (Brown \& Flaming, 1977) established the absolute position in space of the focal plane. The eyecup was then positioned so that the photoreceptors could be penetrated within $25 \mu \mathrm{m}$ above or below the focal plane. 
The signal was recorded on magnetic tape (frequency range d.c. $-1200 \mathrm{~Hz}$ ). After an experiment, the responses were digitized for later analysis.

\section{Light stimulation}

The light from a Xenon lamp (Osram $150 \mathrm{~W}$ ) was split into two beams allowing two stimuli to be varied independently in intensity and position. After the two beams were combined with a prism, the stimuli were imaged on the retina by a Zeiss dissecting microscope (Copenhagen \& Owen, 1976; Copenhagen \& Green, 1985). Both the optical bench and the Faraday cage containing the microscope and the experimental chamber were mounted on an antivibration table (Newport Research Corporation).

\section{Stimulus calibration}

The optical density of the filters was measured in three ways with a calibrated photodiode (Pin 10, United Technology, Inc.), a photomultiplier (American Instrument Co.), and a spectrophotometer (Beckman). Interference filters (510 and $680 \mathrm{~nm}$ ) were used to distinguish between redand green-sensitive cells. The results reported here were obtained from red-sensitive cones using a $650 \mathrm{~nm}$ broad band $(80 \mathrm{~nm}$ half bandwidth) filter. The unattenuated intensity with the broad band filter was equivalent to $1.44 \cdot 10^{7}$ photons $\mathrm{sec}^{-1} \mu \mathrm{m}^{-2}$ at $650 \mathrm{~nm}\left(2.88 \cdot 10^{5}\right.$ photons [20 msec flash $]^{-1} \mu \mathrm{m}^{-2}$ ). Light intensities in this paper are given in logarithmic attenuation of that value.

The distribution of light in the slit image was measured by positioning a $10 \mu \mathrm{m}$ (dia.) pinhole aperture in the focal plane in front of the photomultiplier, and moving the slit across that aperture. The relative intensity of the photomultiplier output for different slit positions is shown in Fig. 1. For comparisons, the calculated convolution of a $5 \mu \mathrm{m}$ wide slit with a $10 \mu \mathrm{m}$ circle is displayed on the same plot. This function describes the magnitude of the photomultiplier output for a diffraction-free, $5 \mu \mathrm{m}$ wide slit moved across a $10 \mu \mathrm{m}$ aperture (dashed line). A comparison of the measured intensity profile with the calculated function provides an estimate of the scattering. The light distribution was measured in planes $25 \mu$ above and below the focal plane. No significant blurring was found. Thus when the photoreceptors were penetrated within $25 \mu \mathrm{m}$ of the focal plane a focused stimulus was flashed on the cones.
We should point out that in spite of these precautions the stimulus is degraded by intraretinal scatter which is inherent to the eyecup preparation.

\section{Experimental procedure}

The microelectrode was advanced through the retina at a $45 \mathrm{deg}$ angle to the vertical, in $4 \mu \mathrm{m}$ steps (nominal speed at each step: $200 \mu \mathrm{m} / \mathrm{sec}$ ) until a photoreceptor was impaled. The hyperpolarizing responses to $510 \mathrm{~nm}$ and $680 \mathrm{~nm}$ light stimuli were compared to distinguish between middle (green) and long (red) wavelength cones. Only the responses from redsensitive cones were analyzed.

Cones were distinguished from horizontal cells by their faster responses, larger depth of recording and smaller receptive fields (Baylor \& Hodgkin, 1973). Red cones were easily distinguished from rods by their higher sensitivity at $680 \mathrm{~nm}$ than at $510 \mathrm{~nm}$. If a cell was identified as a cone, the receptive field space constant was measured. After the slit had been roughly centered (along two perpendicular directions) on the impaled cell, it was displaced laterally and flashed at several positions on each side of the receptive field center and the peak amplitudes of the evoked responses were measured. The fall-off of response amplitude with lateral displacement was adequately described by an exponential decay $(\lambda$, the space constant, varied from 10 to $40 \mu \mathrm{m})$.

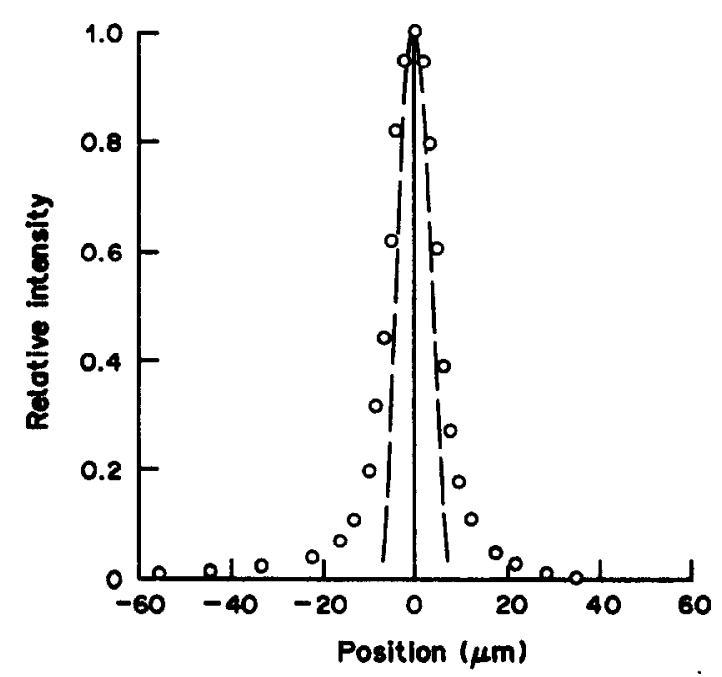

Fig. 1. Light intensity distribution for the slit stimulus. The slit was moved across a $10 \mu \mathrm{m}$ pinhole placed in front of a photomultiplier. The diamonds indicate the amplitude of the photomultiplier output at a series of slit positions. The dushed line indicates the theoretical photomultiplier output for a perfect optical system (the convolution of a $5 \mu \mathrm{m}$ wide slit with a $10 \mu \mathrm{m}$ circular aperture). 
In only six instances did the cone response remain stable (high, constant sensitivity and low noise) long enough to conduct the full set of measurements needed for the excitation analysis. Other measurements were obtained from an additional six cones and are presented in the last section of this paper.

\section{RESULTS}

\section{The basis of the excitation analysis}

Figure 2 illustrates the conceptual framework of the experiment. Two narrow slits $(5 \mu \mathrm{m}$, width) were flashed on the receptive field of the impaled cone. One slit was centered on the impaled cell (intensity noted $I_{c}$ ), and the other (intensity noted $I_{d}$ ) was laterally positioned at a fixed $(30 \mu \mathrm{m})$ distance from the first slit. The slits were flashed either singly or as pairs. The peak amplitudes of the evoked responses were measured.

The process relating the response amplitudes to the slit intensities is shown on Fig. 2. Prior to any spatial interaction, each slit stimulus generates a local neural signal. The amplitude of this signal is related to the slit intensity by the function $e($.$) , called excitation function. The$ excitation generated by the centered slit is noted $E_{c}=e\left(I_{c}\right)$. The excitation generated by the displaced slit in the impaled cone, $E_{d}$, is only a

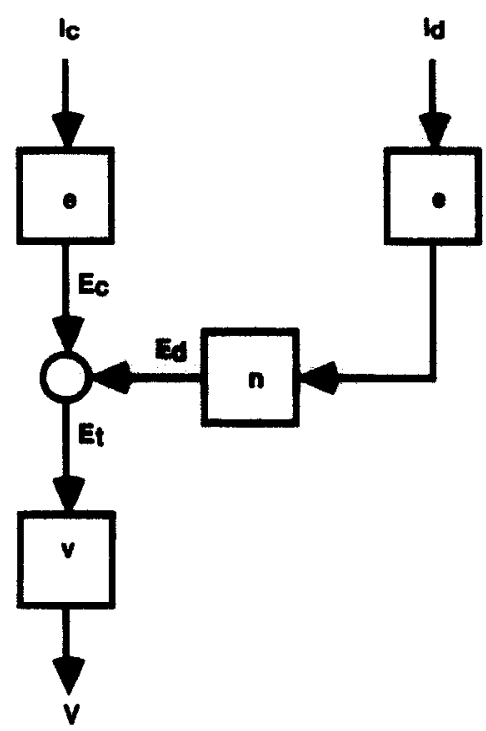

Fig. 2. Diagram to illustrate the relation between stimuli, excitations and response amplitude. Symbols: $\left(I_{s}\right)$ centered slit intensity; $\left(I_{d}\right)$ diepleced slit intencity; $(e)$ excitation function; $\left(E_{r}\right)$ centered alit excitation; $\left(E_{d}\right)$ diplaced sit excitation; $(n)$ coupling function; $\left(E_{t}\right)$ total exitation; $(v)$ late transformation; $(V)$ respones amplitude. See text for details. fraction of the excitation which that slit produced locally. The function $n($.$) accounts for the$ attenuation due to coupling through the network of cones. When the slits are flashed together, the excitations $E_{c}$ and $E_{d}$ add. The total excitation generated in the impaled cone uniquely determines the response amplitude $V=v(E)$. The goal of the excitation analysis is to determine how excitation varies with light intensity.

Figure 3 illustrates for cell number 1 in Table 1 the iterative procedure we used to infer this relationship.

First, centered and displaced slit intensities ( $I_{c}$ and $I_{d}$ respectively) were adjusted to produce responses of equal amplitude $(V)$ (Fig. 3, $\mathrm{A}$ and B: $I_{c}=-1.0 \log$ units, $V=5.8 \mathrm{mV} ; I_{d}=-0.5$ $\log$ units; $V=5.4 \mathrm{mV}$ ). In the conceptual scheme shown in Fig. 2 response amplitude is assumed to be uniquely determined by the total excitation produced in the impaled cone. Thus, independent of the precise relationship between excitation and voltage, equal response amplitudes must be produced by equal excitations.

Next, keeping the intensities unchanged, the two slits were flashed simultaneously and the resulting response amplitude $\left(V^{\prime}\right)$ was measured (Fig. 3c: $V^{\prime}=9.7 \mathrm{mV}$ ). Assuming that the excitation $(E)$ produced by each slit adds linearly (this assumption will be tested later), the total excitation is now $2 E$.

Finally, the centered slit intensity was adjusted until it produced a response $V^{\prime}$. At that intensity, the excitation produced by the slit alone must also be equal to $2 E$. The response in Fig. 3 shows that a 3.2-fold increase in the intensity of the centered slit produced a response whose amplitude ws $8.5 \mathrm{mV}$, slightly less than the $9.7 \mathrm{mV}$ response in Fig. 3C. Thus, about a 4-fold increase of the centered slit intensity is required to produce a 2 -fold increase in excitation.

One can now measure the response amplitude generated by a total excitation of $3 E$ (by simultaneously flashing two slits, one at an intensity known to generate an excitation of $E$, the other at an intensity producing $2 E$ ) and $4 E$ (by simultaneously flashing the two slits at intensities such that they each generate an excitation $2 E$ ). Thus, by systematically increasing intensity and matching individual and paired responses, one can determine the centered slit intensities that produce excitation of $E, 2 E, 3 E \ldots$ In other words, one can measure the excitation function in a discrete fashion. 

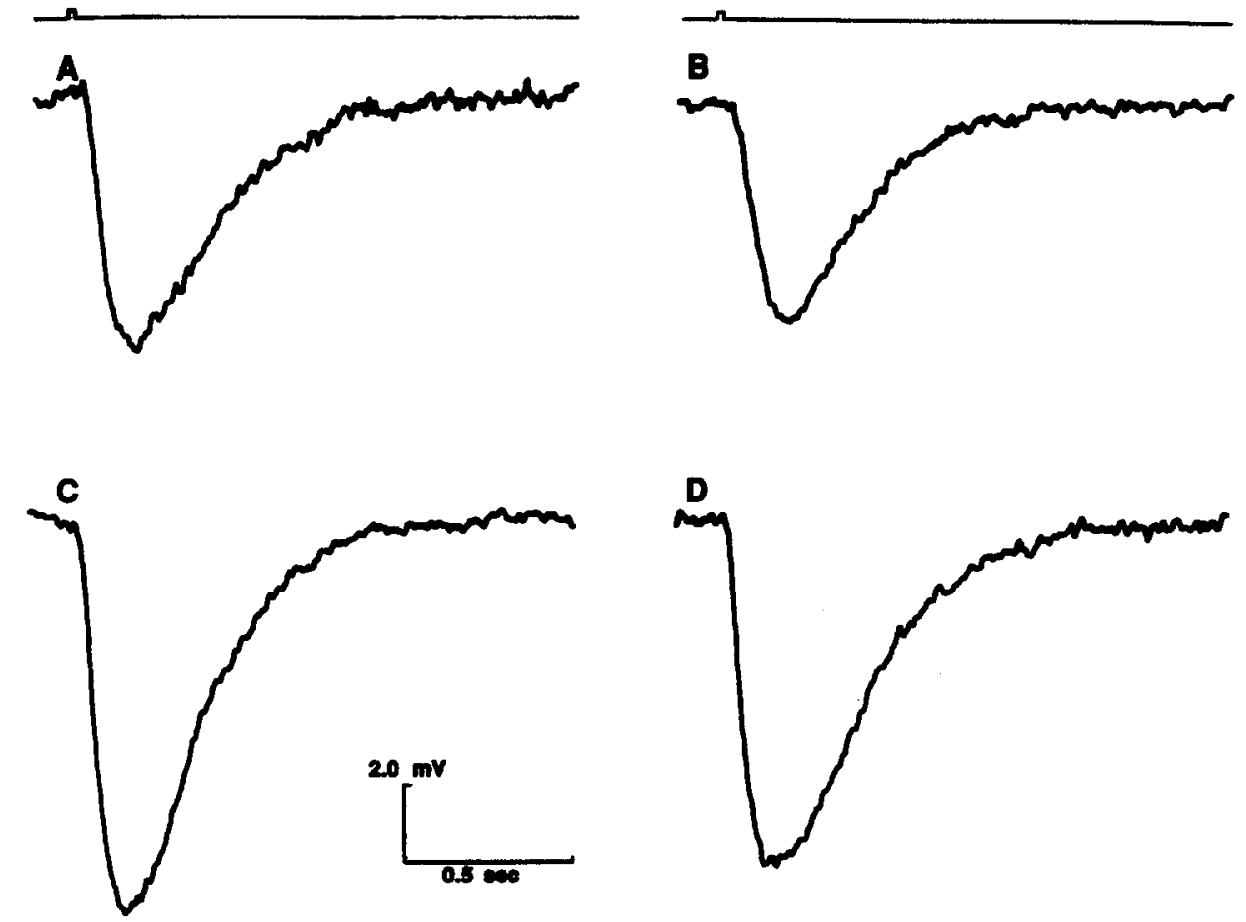

Fig. 3. Slit responses from a cone to illustrate the procedure used to infer how excitation varies with light intensity. (A) Response to a centered slit, $I_{c}=-1.0 \log$ units. (B) Response to a displaced slit, $30 \mu \mathrm{m}$ away from the receptive field center, $I_{d}=-0.5 \mathrm{log}$ units. Note the response is about equal to that in $A$. (C) The two stimuli "A and B" flashed together 20 as to produce an excitation of $2 E$. (D) Centered slit increased in intensity to approximately double excitation, $I_{c}=-0.5 \log$ units (same cell as in Table 1, cell no. 1).

Inferring the excitation function from the intensity-response curves

Adjusting intensities to match response amplitudes can be tedious and impractical given the short lifetime of the intracellular penetration. Instead we first measured intensity-response curves separately for the centered and the displaced slit (Fig. 4A). Next, both slits were flashed simultaneously. While the intensity of the centered slit was kept constant, the intensity of the other was systematically increased over the full intensity range. The process was then repeated, with the intensity of the centered slit fixed at another value, and so on until responses to a complete set of intensity pairs were obtained (Fig. 4A). During this process, the response to a fixed-intensity center stimulus was periodically measured in order to ensure that the sensitivity of the cell had remained constant.

Response amplitudes for any combination of intensities were obtained from the intensityresponse curves by linear interpolation between points. The first iteration of the procedure is illustrated by the dashed lines in Fig. 4. Since the excitation variable is operationally defined, it is measured on an arbitrary scale. The data point marked " $A$ " in Fig. $4 A$ is arbitrarily chosen to define $I_{c, 1}$, the centered slit intensity which evokes an excitation $E=1$. (The subscripts indicate the slit position and the evoked excitation.) The corresponding response amplitude is $V_{1}=1.6 \mathrm{mV}$. The intensity of the displaced slit $I_{d, 1}$ which evokes the same excitation $E=1$ is determined by the intersection " $B$ " of the constant amplitude passing through " $A$ " and the linear interpolation line joining the displaced slit data points. From Fig. 4B, we can find " $C$ ", the response amplitude $V_{2}$ to the pair of slits flashed at intensities $I_{c, 1}=-2.0$ and $I_{d, 1}=-1.4 \log$ units (for some other pairs of intensities an additional interpolation between two curves of constant centered slit intensity was required). This response amplitude corresponds to an excitation $E=2$ and therefore, the intensity $I_{c, 2}$ can now be obtained from Fig. 4A (point " $D$ "). By following the same procedure iteratively, the intensities $I_{c, 1}, I_{c, 2}, I_{c, 3}, \ldots$ and $I_{d, 1}, I_{d, 2}, I_{d, 3}, \ldots$ producing $E=1,2$, $3, \ldots$ (respectively) were measured from the intensity-response curves. 


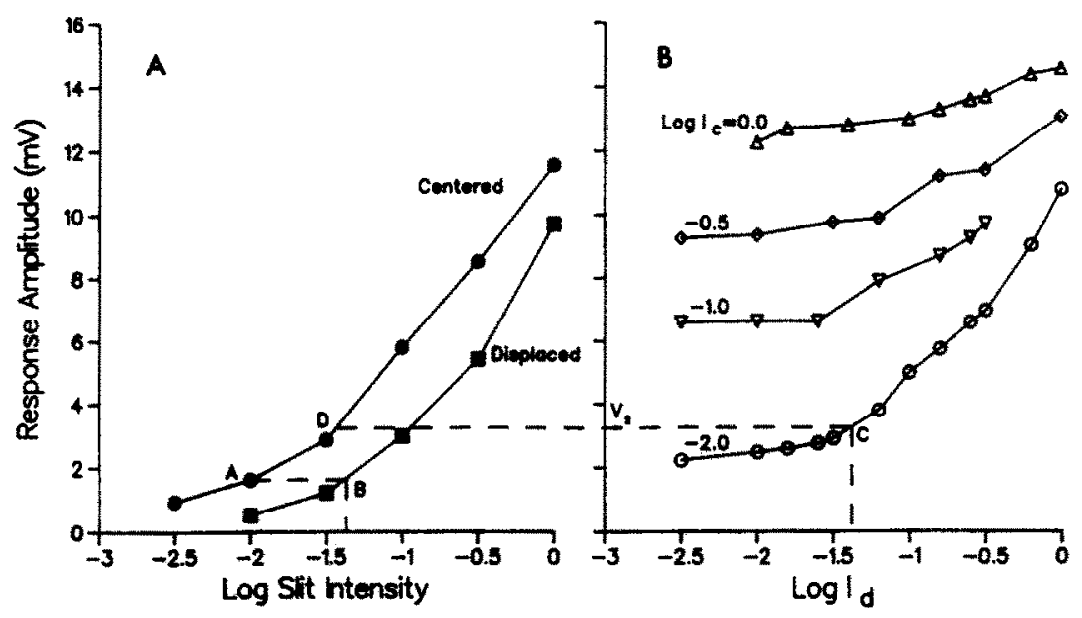

Fig. 4. (A) Response peak amplitudes for the centered and the displaced slit flashed singly. (B) Response amplitude for the two slits thahed simultaneously. The abscissa indicates the displaced slit intensity. Each set of data comes from pairing a displaced slit of varizble intensity with a fixed centered slit of fixed intensity. See the text for an explanation of the dashed lines (same cell as in Fig. 3).

Thus the excitation function $E=e\left(I_{c}\right)$ was measured for integer values of $E$. As seen in Fig. 5, when plotted on logarithmic coordinates, it is well fitted by a straight line over approx. 2 $\log$ units of intensity (slope $=0.45$, regression coefficient $r^{2}=0.995$ ). As seen in Table 1, other cells produced almost identical results. The range over which the excitation function was measured varied from 1.5 to $2.0 \log$ units of intensity. Over that range, excitation is a compressive power law of the slit intensity (average exponent: $0.48, n=5$ ):

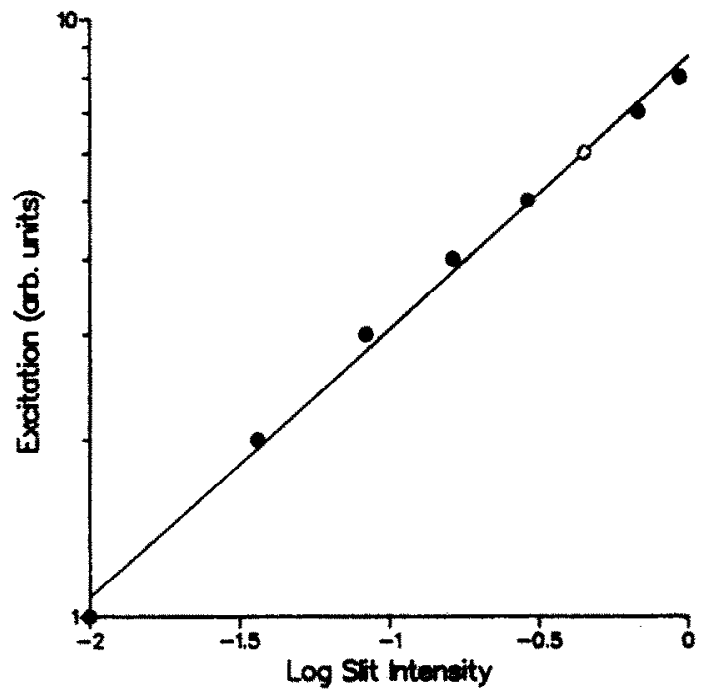

Fig. 5. Excitation function obtained from the cone in Figs 3 and 4 with slit stimuli. The abscien indicaties the intencity of the centered slit, and the ordinate gives the evoked excitation. The solid circles were inferred from the data shown in Fig. 4. The straight line is a regresion line fitted to the discrete excitation values (alope $=0.48, r^{2}=0.995$ ) on the logarithmic axes.

$$
E=k \cdot r^{0.5} .
$$

To test whether this result was dependent on the shape of the stimulus, the experiment was also conducted using two small spots $(7 \mu \mathrm{m}$ in dia.). The excitation function for these stimuli was also well fitted by a compressive power law (see Fig. 6).

\section{Test of the linear addition of the excitations}

In order to compute the excitation function, the procedure presented above assumes that the excitations add linearly so that the total excitation produced by two slits flashed simultaneously is simply the algebraic sum of the excitations they produce alone. It is possible to test the validity of this assumption. The reasoning goes as follows: when the central slit at intensity $I_{c}$ and displaced slit at intensity $I_{d}$ individually produce a response of amplitude $R_{1}$ the excitation evoked is assumed to be equal (say $E_{1}$ ). Likewise, if $I_{c}^{\prime}$ and $I_{d}^{\prime}$ produce a response of amplitude $R_{2}$ the excitation is assumed to be $E_{2}$. Linear addition requires

Table 1. Summary of the excitation analyses. The columns labelled $\lambda^{+}$and $\lambda^{-}$give the space conetenus for the exponential function which fitted the date points on oppolte aldes of the receptive fidd. The $\log I$ colpmi give the butenity range over which the excitation function whe futed th power low, $m$ is the exponent and $r^{2}$ is the remprion coeflicient for the power bw which eve the but it

\begin{tabular}{cccccc}
\hline Cell no. & $\lambda^{+}(\mu \mathrm{m})$ & $\lambda^{-}(\mu \mathrm{m})$ & $\log I$ & $m$ & $r^{2}$ \\
\hline 1 & 27 & 34 & 2.0 & 0.45 & 0.95 \\
2 & 19 & 24 & 1.6 & 0.51 & 0.98 \\
3 & 26 & 29 & 1.5 & 0.5 & 0.98 \\
4 & 29 & 25 & 1.7 & 0.48 & 0.99 \\
5 & 32 & 23 & 2.0 & 0.46 & 0.996 \\
\hline
\end{tabular}




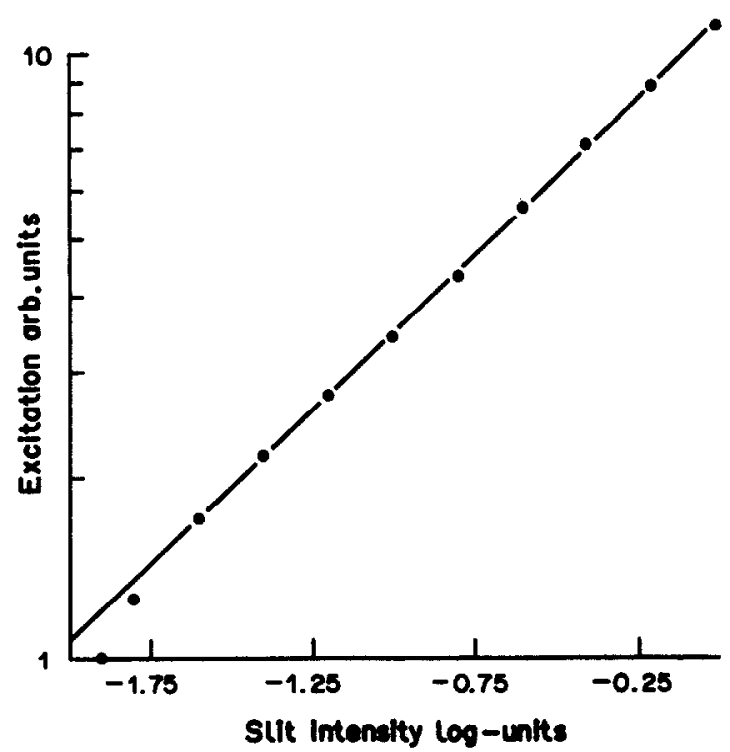

Fig. 6. Excitation function obtained from a cone by using two small spot $(5 \mu \mathrm{m}$ dia.) stimuli. The straight line was fitted to the inferred excitation points (O) (slope $=0.51$, regression $r^{2}=0.995$ )

equal responses from combinations of spatially separated stimuli having the same total excitation. That is, $\left(E_{1}+E_{2}\right)$ is the excitation from both $\left(I_{c}+I_{d}^{\prime}\right)$ and $\left(I_{c}^{\prime}+I_{d}\right)$ and consequently these stimuli should produce responses of the same amplitude. This should be true, independent of the form of the transduction function. (The excitations resulting from the stimuli $\left(I_{c}+I_{c}^{\prime}\right)$ and $\left(I_{d}+I_{d}^{\prime}\right)$ would be $\left(E_{1}+E_{2}\right)$ only if the transduction function itself were linear.)

Centered slit intensities and displaced slit intensities that produce the same excitation were obtained from data such as shown in Fig. 4A. Horizontal lines were drawn through the data points and the pair of intensities corresponding to the same excitation (since by construction the response amplitudes were equal) were formed. Linear interpolation was used when data points for the two stimuli did not correspond to equal response amplitude. Using Fig. 4B, we determined the response amplitudes for the pairs of composite stimuli leading to the same excitation (again linear interpolation was used when necessary). For each composite excitation, we thus measured two response amplitudes, which according to the assumption of the excitation analysis, should be equal. We plotted one against the other and looked for deviation from a line of unit slope through origin. This analysis was performed on the data from 5 units. In each case, results similar to those in Fig. 7 were obtained. The deviations from the $\mathbf{4 5} \mathrm{deg}$ line were small and thus support the excitation

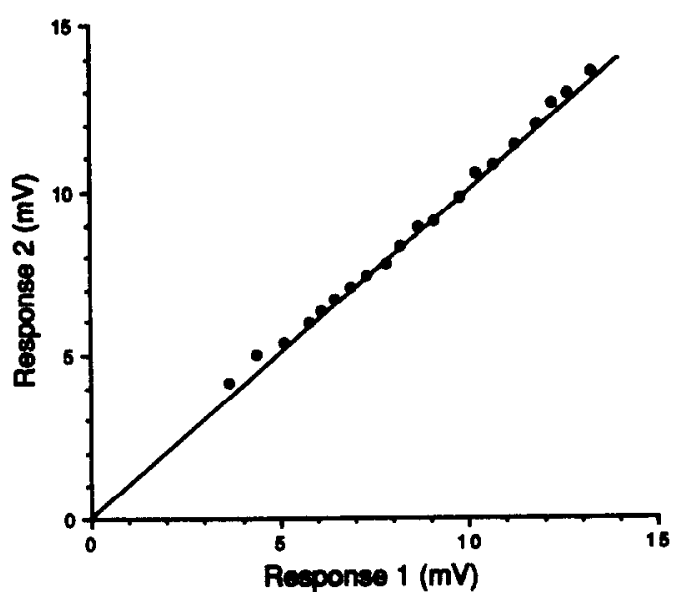

Fig. 7. Test of linear addition of the excitations. Each point shows responses from combinations of intensities which produce the same total excitation. The response amplitudes were measured from the intensity-response curves shown in Fig. 4. The ordinate axis corresponds to response amplitudes obtained when the displaced slit intensity was higher than that of the centered alit and vice vera for the abacisa axis. Linear addition requires that equal responses will be evoked by identical amounts of total excitation. Thus the extent to which the points fall on a 45 deg line is a verification of the hypothesis of linear addition.

analysis. In four of the six cells analyzed, we noticed a slight bias of the data points toward the upper side of the $45 \mathrm{deg}$ line. We do not know whether such a small bias is of any significance.

\section{Response and sensitivity receptive fields}

From the excitation analysis, we concluded that the excitation generated locally by a small stimulus is proportional to the square root of its intensity. If the excitation function truly describes a local mechanism, the results from the excitation anlaysis should be valid for other slit displacements. For small responses both $v($.) and $n($.$) (not shown here; see Pluvinage \&$ Green, 1990) are reasonably linear. The following approximation should then hold:

$$
V(x) \cong k(x) \cdot I^{m} ;
$$

where $x$ is the distance between the slit and the center of the receptive field.

Equation (2) can be tested experimentally. The spatial weighting function $k(x)$ is measured by flashing a slit of fixed intensity at several positions on the receptive field. Since the amplitude of the response decays exponentially with distance:

$$
k(x) \propto e^{-|x| / i_{r}}
$$

where $\lambda_{r}$ is the response space constant. 


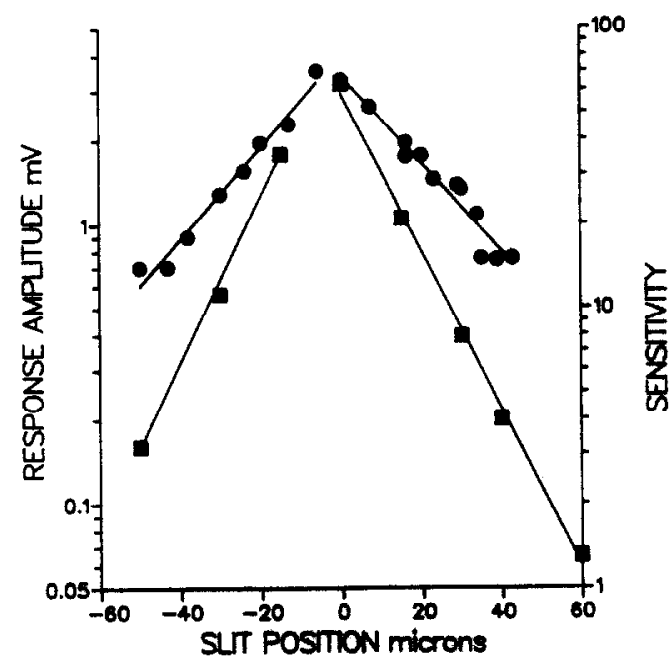

Fig. 8. Comparison between reaponse and sensitivity receptive field profiles (cell no. 9). (O) Reeponee amplitude to a fixed intensity slit $(-1.8 \mathrm{log}$ units). The solid lines were fitted by regression (speces constants: $\lambda_{r-}=26 \mu \mathrm{m}$, $\lambda_{r+}=28 \mu \mathrm{m}$ ). (O) Sensitivity meanurements (fixed response amplitude: $3 \mathrm{mV}$ ). Sensitivity is defined to be the ratio of the threshold intensity ( $3 \mathrm{mV}$ criterion) to the full intensity. The lines, fitted by regression, correspond to sensitivity space constants of $\lambda_{s-}=14.5 \mu \mathrm{m}$ and $\lambda_{s+}=15.5 \mu \mathrm{m}$.

Next, the intensity needed to produce a given response amplitude (fixed $V$ ) is measured at several positions. Adjusting the slit intensity to produce a given response amplitude $V$ should lead to (using equations 2 and 3):

$S(x)=1 / I(x)=V^{-1 / m} \cdot[k(x)]^{1 / m} \propto e^{-|x| /\left(m i_{1}\right)}$;

where $S(x)$ is the sensitivity and $I(x)$ is the intensity needed at position $x$ to evoke a criterion response amplitude $V$. Thus $\lambda_{s}$, the space constant for the fall-off in sensitivity, and the response space constant should be related by:

$$
\lambda_{s}=\lambda_{r} \cdot m
$$

Figure 8 presents typical results from a cone on which the receptive field profile was obtained by measuring $V(x)$ and $S(x)$ at several slit positions. The response receptive field profile $[V(x), 0]$ was fitted by exponential decays with the space constants $\lambda_{r-}=26 \mu \mathrm{m}$ for negative displacements and $\lambda_{r^{+}}=28 \mu \mathrm{m}$ for positive slit displacements (the inverse of the slopes' regression lines). The sensitivity receptive field profile $[S(x), D]$ also decays exponentially, but the space constants are $\lambda_{s-}=14.5 \mu \mathrm{m}$ and $\lambda_{s^{+}}=15.5 \mu \mathrm{m}$. The average ratio, $\lambda_{g} / \lambda_{r}$, of the sensitivity to response space constant on each side of the receptive field center is $\mathbf{0 . 5 4}$.

Table 2 gives the response and sensitivity space constants measured on six cells. Thus, for
Table 2. Comparison between response and sensitivity space constants from the receptive fields of six cells. The superscripts " -" and "+" indicate the side of the receptive field (negative or positive displacements respectively). The subscripts " $r$ " and " $s$ " relate to response and sensitivity measurements respectively. The entries in the last column

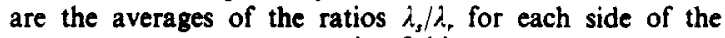
receptive field

\begin{tabular}{cccccc}
\hline Cell no. & $\lambda_{r}^{-}$ & $\lambda_{r}^{+}$ & $\lambda_{s}^{-}$ & $\lambda_{s}^{+}$ & $\lambda_{s} / \lambda_{r}$ \\
\hline 1 & 27 & 34 & 15 & 18 & 0.55 \\
6 & 18 & 22 & 12 & 14 & 0.64 \\
7 & 25 & 22 & 14 & 13 & 0.58 \\
8 & 36 & 26 & 19 & 13 & 0.52 \\
9 & 26 & 28 & 14 & 15 & 0.54 \\
10 & 29 & 33 & 17 & 15 & 0.52 \\
\hline
\end{tabular}

the cell in Fig. 8 and five others sensitivity decreases with slit displacement twice as fast as the response to a fixed intensity slit, a result consistent with an early square-root transformation. One cell (not shown in Table 2) had a very steep receptive field, which was not adequately fitted by exponential fall-off, and the response and sensitivity receptive fields were similar. The results for this cell are presented and analyzed in detail in the second paper (Pluvinage \& Green, 1990).

\section{DISCUSSION}

The experiments presented here give support to the notion that a square root transformation occurs at the photoreceptor level. How can this occur given that previous studies have shown that both photocurrent and full-field photovoltage are Michaelis-Menten functions of light intensity? One possibility is that one of the assumptions underlying the excitation analysis is incorrect. These assumptions are: (1) the inferred excitation function describes mechanisms before the spatial integration of the excitatory signals; (2) the slits stimulate independent sets of receptors; (3) the excitatory signals add linearly; (4) the responses are adequately characterized by their peak amplitude. Let us examine each of them in detail.

To satisfy assumption (1) small spots might seem to be the best stimuli, but we usually used long narrow slits. Each slit must actually stimulate a row of photoreceptors. Could the square root transformation we inferred result from the integration of the signals produced along the slit? Results from previous studies (Lamb \& Simon, 1976) are consistent with the notion that, with a slit stimulus, there is no gradient in the longitudinal direction. That is, a slit "isolates" the effect of coupling along the direction 
perpendicular to the slit, which allows us to measure the local transformation prior to the spatial integration of signals. Direct evidence of that point comes from the excitation analysis conducted using two small spots of light. As shown in Fig. 6, a square root function also describes the small spot data, a result consistent with the assumption that we are probing a transduction mechanism prior to the spatial integration of excitatory signal.

One of the basic assumptions of the excitation analysis, as illustrated in Fig. 2, is that the slits act on independent sets of photoreceptors. What about light scatter? If the peripheral slit acted solely through scattered light then the procedure used to infer the excitation function should always lead to the conclusion that exci-
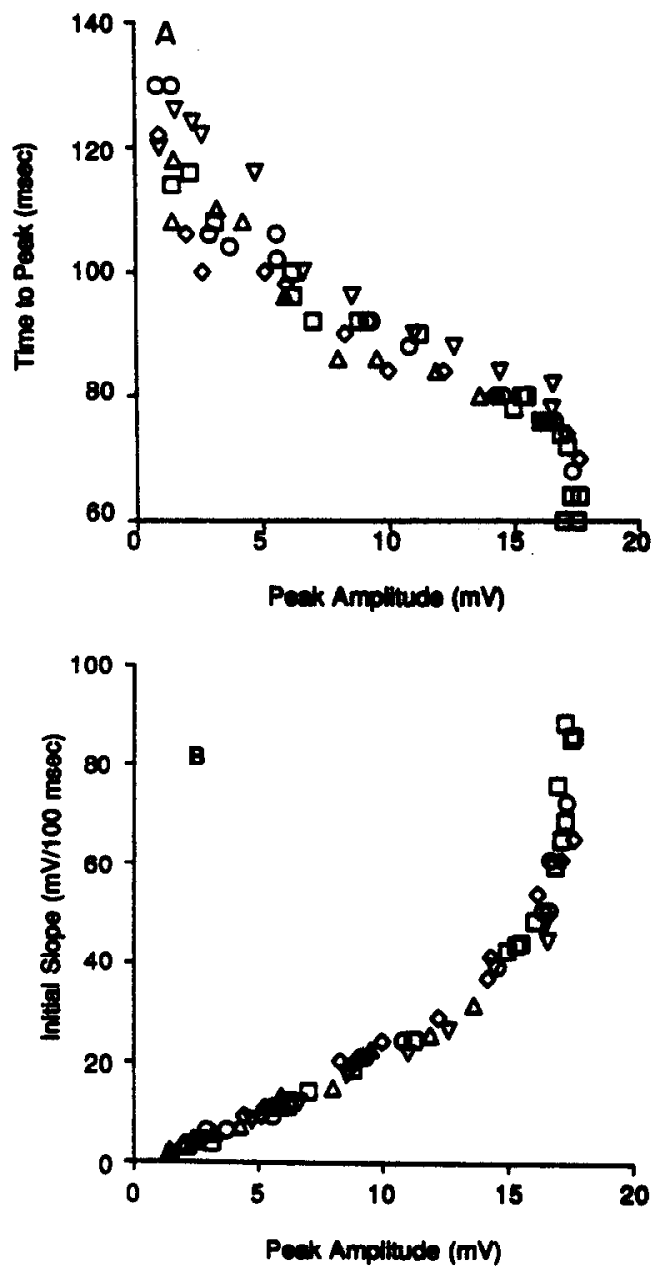

Fig. 9. (A) The relationship between response time-to-peak and the peak amplitude is indicated for stimuli of different intensities and positions for one cone: $(\square)$ full-field; slit, $(\Delta)$ position $+23 \mu \mathrm{m}$; (O) position $+10 \mu \mathrm{m}$; (O) position $-5 \mu \mathrm{m} ;(\nabla)$ position $-17 \mu \mathrm{m}$. (B) The relationship between the initial slope and the peak amplitude is indicated for various stimuli for the same cone. tation grows linearly with light intensity. This is because: (a) equating the responses to slits should equate the quantum catch in the impaled cone; (b) flashing the two slits simultaneously should result in a linear addition of the light intensities prior to the transduction function; and (c) the response produced by equated slits flashed as a pair should be matched by flashing either slit at twice the intensity. Thus, whenever one infers a highly nonlinear transduction function as we do the two slits must to a large degree be acting independently. Further evidence against scatter comes from the excitation experiments. The total excitation due to two slits would not be equal to the sum of the individual excitations unless the transduction function was also linear, an unlikely possibility over the wide range of intensities we tested. Thus the test for linear addition (Fig. 7) should have failed and it did not. On the basis of the linear addition shown in Fig. 7 one can conclude that the images of $30 \mu \mathrm{m}$ separated slit stimuli are largely nonoverlapping. Any overlap must be so slight that it is insufficient to lead to a clear failure of the excitation addition test.

For the simplicity of our analysis the responses were characterized using a single parameter, the peak amplitude. However, the dynamics of the response depends on both the position and the intensity of the stimulus. Could neglecting this be the problem? Figures $9 \mathrm{~A}$ and B show data obtained in an attempt to address this issue. To a reasonable approximation, independent of the stimulus size, position or intensity, responses of similar amplitude have similar time-to-peak and initial slope. Thus, when the peak amplitude of two responses are equal, the early part of the responses (up to the peak) should also coincide. But when two slits that evoke very different response amplitudes are flashed simultaneously, the excitatory signals from each slit are likely to peak at different times, a complexity not included in our analysis. Moreover, responses to intense stimuli typically exhibit a "plateau" after the peak which is absent from weak responses. Nonetheless, the inferred power law excitation function correctly predicted that, with slits, the sensitivity and the response receptive field would decay exponentially with space constants in a ratio $1: 2$.

In this regard it should be noted that there were small but consistent discrepancies between the data and the predictions of equations (2-5). For example, the excitation analysis inferred an 
average power law exponent of $m=0.51$ but the average ratio of sensitivity to response space constant is $\lambda_{s} / \lambda_{r}=0.56$. This probably represents more than experimental error since on cell no. 1 (the only cell on which both experiments were conducted) there was a similar difference ( $\left.m=0.45, \lambda_{s} / \lambda_{r}=0.55\right)$. This second order effect is examined by the model presented in the paper which follows (Pluvinage \& Green, 1990).

What about the apparent conflict between Michaelis-Menten relationships and the inferred square root law? Suction electrode photocurrent measurements predominantly reflect the properties of a single cell (Baylor, Lamb \& Yau, 1979). Full-field stimuli should functionally uncouple the impaled cone from its neighbors since no current flows between identically responding cones. Thus, in both cases, the physiological responses are measured in absence of interreceptor coupling. On the other hand, in our experiments with slit and spot stimuli cone coupling significantly affects response amplitudes. In Pluvinage and Green (1990) experimental evidence is presented which shows that the square root law depends on coupling between cones and that it arises because of local saturation and recruitment of scattered light responses.

Acknowledgements - This study was supported by NIH grant EY00379. The authors are indebted to David Krantz and Stephen Easter for helpful diseussions and to Marilyn Glover for her assistance in the analysis of data and in the preparation of the manuscript.

\section{REFERENCES}

Baylor, D. A. \& Hodgkin, A. L. (1973). Detection and resolution of visual stimuli by turtie photoreceptors. Journal of Physiology, London, 234, 163-198.

Baylor, D. A., Fuortes, M. G. F. \& O'Bryan, P. M. (1971). Receptive fields of cones in the retina of the turtle. Journal of Physiology, London, 214, 265-294.

Baylor, D. A., Hodgkin, A. L. \& Lamb, T. D. (1974). The electrical response of turtle cones to flashes and steps of light. Joumal of Physiology, London, 242, 685-727.

Baylor, D. A., Lamb, T. D. Yau, K.-W. (1979). The membrane current of single rod outer semgents. Journal of Physiology, London, 288, 589-611.
Brown, K. T. \& Flaming, D. G. (1977). New microelectrode techniques for intracellular work in small cells. Neuroscience, 2, 813-827.

Copenhagen, D. R. \& Green, D. G. (1985). The absence of spread of adaptation between rod photoreceptors in turtle retina. Journal of Physiology, London, 369, 161-181.

Copenhagen, D. R. \& Owen, W. G. (1976). Functional characteristics of lateral interactions between rods in the retina of the snapping turtle. Journal of Physiology, London, 259, 251-282.

Debreu, G. (1959). "Topological methods in cardinal utility theory." In Arrow, K. J., Karlin, S. \& Suppes, P. (Eds.), Mathematical methods in the social sciences. Stanford, C. A.: Stanford University Press.

Easter, S. S. (1968). Excitation in the goldfish retina evidence: for a non-linear intensity code. Journal of Physiology, London, 195, 253-271.

Enroth-Cugell, C. \& Harding, T. H. (1980). Summation of rod signals within the receptive field centre of cat retinal ganglion cells. Journal of Physiology, London, 298, 235-250.

Grusser, O. J. (1971). A quantitative analysis of spatial summation of excitation and inhibition within the receptive field of retinal ganglion cells of cats. Vision Research, 3, 103-127.

Krantz, D. H. (1973). Measurement-free tests of linearity in biological systems. IEEE Transactions on Systems, Man \& Cybernetics, 3, 266-271.

Krantz, D. H., Luce, R. D., Suppes, P. \& Trersky, A. (1971). In Foundations of mearurement (Vol. 1). London: Academic Press.

Kruskal, J. B. (1965). Analysis of factorial experiments by estimating monotone transformations of the data. Journal of the Royal Statistics Society (Series B), 27, 251-263.

Lamb, T. D. \& Simon, E. J. (1976). The relation between intercellular coupling and electrical noise in turtie photoreceptors. Journal of Physialogy, London, 263, 257-286.

Levine, M. W. \& Abramov, I. A. (1975), An analysis of spatial summation in the receptive fields of goldfish retinal ganglion cells. Vision Research, 15, 777-789.

Pluvinage, V. \& Green, D. G. (1990). Square root intensity coding in turtle cones: Physiological mechanisms. Vision Research, 30, 683-691.

Schnapf, J. L. \& McBurney, R. N. (1980). Light-induced changes in membrane current in cone outer segments of tiger salamander and turtle. Nature, London, 287, 239-241.

Stevens, S. S. (1970). Neural events and the psychophysical law. Science, New York, $170,1043-1050$.

Stone, J. \& Fabian, M. (1968). Summing properties of the cat's retinal ganglion cell. Vision Research, 8, 1023-1040. 